\title{
Médiévales
}

Langues, Textes, Histoire

59 | automne 2010

Théâtres du Moyen Âge

\section{Vivre une métaphore : écritures anglo-saxonnes du voyage en mer au VIII ${ }^{\mathrm{e}}$ siècle}

Living a Metaphor : Anglo-Saxon Writings about See Travels in the Eighth

Century

\section{Guénolé Ridoux}

\section{(2) OpenEdition}

\section{Journals}

Édition électronique

URL : https://journals.openedition.org/medievales/6140

DOI : 10.4000/medievales.6140

ISSN : 1777-5892

Éditeur

Presses universitaires de Vincennes

Édition imprimée

Date de publication : 31 décembre 2010

Pagination : 145-169

ISBN : 978-2-84292-267-2

ISSN : 0751-2708

Référence électronique

Guénolé Ridoux, "Vivre une métaphore : écritures anglo-saxonnes du voyage en mer au vIII siècle », Médiévales [En ligne], 59 | automne 2010, mis en ligne le 01 juin 2012, consulté le 22 avril 2022. URL: http://journals.openedition.org/medievales/6140; DOI : https://doi.org/10.4000/medievales.6140 
Médiévales 59, automne 2010, p. 145-169

Guénolé RIDOUX

\section{VIVRE UNE MÉTAPHORE : ÉCRITURES ANGLO-SAXONNES DU VOYAGE EN MER AU VIII' ${ }^{\mathrm{e}}$ SIÈCLE}

Les historiens contemporains ont beaucoup insisté sur la nécessité d'adapter la conception moderne du pèlerinage au contexte de la culture religieuse de l'antiquité tardive et du début du Moyen Âge ${ }^{1}$. S'il est d'usage de distinguer les voyages à destination des lieux saints (peregrinationes ad loca sancta) de ceux à propos desquels l'acte de voyager semble l'emporter sur l'intérêt pour les lieux de culte (ex patria ou pro Domino $)^{2}$, on a pu rappeler que ces deux formes du mouvement religieux n'étaient pas entièrement étanches $^{3}$. Pour mieux rendre compte de l'idéal du voyage durant cette période, il est apparu nécessaire de replacer son étude dans le cadre plus large de l'histoire du monachisme ${ }^{4}$. Des auteurs se sont attachés à montrer qu'à une époque où l'expression des aspirations ascétiques était encore peu encadrée, le mouvement physique, l'errance, était une forme légitime et répandue de la vie de

1. Cet article reprend des réflexions élaborées au cours d'un travail de recherche mené sous la direction de Stéphane Lebecq (Missions et pérégrinations : des religieux anglo-saxons sur la route, Mémoire de $2^{\mathrm{e}}$ année de master, Université Charles-de-Gaulle Lille 3, 2008). Toute ma reconnaissance va à Stéphane Lebecq et à Charles Mériaux pour leur précieux soutien lors de mes recherches et à Alban Gautier qui a accepté de relire cet article.

2. Ainsi D. WebB, Pilgrims and Pilgrimage in the Medieval West, Londres, 1999, p. 7-8.

3. P. Maraval, Lieux saints et pèlerinages d'Orient, Paris, 2004, p. 11 ; M. Dietz, Wandering Monks, Virgins and Pilgrims, University Park (Penn.), 2005, p. 43-68; G. Constable, « Monachisme et pèlerinage au Moyen Âge », Revue historique, 258, 1977, p. 3-27, sp. p. 13.

4. A. Guillaumont, « Le dépaysement comme forme d'ascèse dans le monachisme ancien », [1968], rééd. dans ID., Aux origines du monachisme chrétien, Bégrolles-en-Mauges, 1979, p. 89-116; J.-M. SANSTERRE, «Attitude à l'égard de l'errance monastique en Occident du $\mathrm{VI}^{\mathrm{e}}$ au XI $\mathrm{XI}^{\mathrm{e}}$ siècle », dans A. Dierkens et ID. dir., Voyages et voyageurs à Byzance et en Occident, Genève, 2000, p. 215-234 ; G. Constable, « Monachisme et pèlerinage... », loc. cit. 
moine ${ }^{5}$. Le processus de définition d'un modèle monastique cénobite, l'insistance croissante des Pères de l'Église sur le devoir d'obéissance et d'autosuffisance, marginalisèrent ce type de dévotion. Cette exclusion progressive, sous la pression des autorités ecclésiastiques et séculières, culmina peut-être, en Occident, avec la diffusion de la Règle de saint Benoît et du principe de stabilité qu'elle soutenait.

Nous consacrerons cet article à l'idéal de l'exil tel qu'il apparaît dans un groupe de textes produits par des auteurs anglo-saxons dans le siècle qui précède la décision prise en 817 au concile d'Aix-la-Chapelle d'imposer la Règle bénédictine aux monastères de l'Empire ${ }^{6}$. Cet idéal met en scène l'aspiration chrétienne au détachement du monde, exprimée au moyen d'images empruntées à la tradition, sans trop d'égards pour les motivations concrètes des voyages et leurs destinations. La persistance de ce motif littéraire dans ces sources doit surprendre l'historien. Ce topos hagiographique ne se développa pas, ici, à la marge des cercles bénédictins, mais fut au contraire mis en œuvre par des auteurs qui insistaient volontiers sur la participation des personnages dont ils rapportaient la geste à la diffusion de la Règle sur leur île natale et sur le continent ${ }^{7}$. Comme nous le verrons, les exilés et leurs biographes connaissaient les objections à l'errance, auxquelles ils se rallièrent parfois. Du point de vue de la forme des voyages, le caractère organisé des missions continentales anglo-saxonnes a souvent été opposé au caractère plus spontané et mobile des voyages irlandais ${ }^{8}$. Au niveau littéraire, l'insistance des auteurs sur l'activité missionnaire des saints, sur leurs qualités toutes « bénédictines » a été interprétée comme autant de moyens employés pour atténuer le thème de l'aspiration ascétique au mouvement qui ne pouvait manquer d'échapper au public, cénobite, auquel ces textes étaient destinés 9 .

Nous nous efforcerons de montrer que, pour les auteurs du « corpus missionnaire », le déplacement physique demeure, comme chez leurs prédé-

5. M. DieTZ, op. cit. ; D. CANer, Wandering, Begging Monks : Spiritual Authority and the Promotion of Monasticism in Late Antiquity, Berkeley, 2002.

6. Sur la chronologie des voyages religieux et leur rapport avec la Règle : J.-M. SANSTERRE, «Attitude à l'égard de l'errance... », loc. cit., p. 224 ; G. Constable, « Monachisme et pèlerinage... », loc. cit., p. 8-14 ; M. DIETZ, op. cit., p. 213-220.

7. Lettre 86 (Boniface) : Die Briefe des Heiligens Bonifatius und Lullus, M. TANGL éd., MGH Ep. select., 1, Berlin, 1916, p. 193 (dorénavant abrégé en : Lettre, numéro de la lettre, nom de l'auteur entre parenthèses, page) ; STÉPHANE DE RiPOn, Vita Wilfridi, ch. 14, B. Colgrave éd., The Life of Bishop Wilfrid by Eddius Stephanus, Cambridge, 1927, rééd. 1985, p. 30.

8. K. Hughes, « The Changing Theory and Practice of Irish Pilgrimage », The Journal of Ecclesiastical History, 9/2, p. 143-151, en particulier. p. 145; G. Constable, « Monachisme et pèlerinage... », loc. cit., p. 10.

9. Cf. M. Dietz, Wandering Monks..., loc. cit., p. 200-212. 
cesseurs latins ou grecs, la mise en pratique d'une métaphore. Le contexte particulier dans lequel ils produisent leurs récits, la nécessité pressante de justifier les voyages qu'ils relatent, les poussent à insister sur le caractère intentionnel de l'exil au monde par le mouvement et à chercher, dans les conditions matérielles de l'exil, des faits concrets qui répondent à l'imaginaire de l'homo viator. La place nécessaire à l'expression de la «métaphore vécue », controversée, se trouve ainsi libérée par l'existence de l'espace géographique bien réel qu'est celui des mers du Nord. Cette emphase sur le milieu marin, frontière et lieu d'errance, permet, à son tour, de donner une consistance originale à la "patrie abandonnée », thème qui est, depuis longtemps, un lieu commun de l'hagiographie voyageuse.

Nous nous appuierons au cours de cette étude sur les premières «Vies missionnaires » du corpus décrit par Ian Wood ${ }^{10}$. Ces textes ont été rédigés dans les scriptoria de Germanie et de Gaule par des religieux d'origine insulaire qui avaient eux-mêmes pris le chemin de l'exil. Ils se divisent en deux " familles " ${ }^{11}$; l'une est liée à saint Boniface et au siège épiscopal de Mayence, l'autre à saint Willibrord et au monastère d'Echternach. Le corpus s'ouvre avec la Vie de Boniface par Willibald, rédigée dans les années $760^{12}$. L'auteur, proche du clergé mayençais, s'est abondamment inspiré de la correspondance du personnage dont il écrit l'histoire. La Vie de Boniface sert à son tour de modèle aux Vies des deux frères Willibald (qui ne doit pas être confondu avec l'hagiographe du même nom) et Wynnebald par Hugeburc ${ }^{13}$, religieuse d'un monastère fondé par Wynnebald en Germanie. Ces récits furent produits dans la deuxième moitié du vIII ${ }^{\mathrm{e}}$ siècle $^{14}$. La seconde famille de textes s'articule autour de la Vie de Willibrord par Alcuin ${ }^{15}$, écrite dans les années 790, seul texte du groupe « willibrordien » dont nous puissions affirmer avec certitude que son auteur était lui-même anglo-saxon. En plus de ces vitae qui appartiennent, à strictement parler, au corpus missionnaire, nous nous intéresserons à deux autres sources, produites quelques années auparavant en Bretagne, qui les ont influencées. L'auteur de la Vita Bonifatii ne connaissait pas l'Histoire ecclésiastique de Bède, dont la première version a pourtant été rédi-

10. I. N. Wood, The Missionary Life : Saints and the Evangelization of Europe, 400-1050, Harlow, 2001 ; J. T. PALMER, Anglo-Saxons in a Frankish World, Turnhout, 2009.

11. Cf. Fig. 1.

12. Willibald, Vita Bonifatii, W. Levison éd., MGH SRG, 57, Hanovre, 1905 (BHL n 1400).

13. Hugeburc, Vitae Willibaldi et Wynnebaldi auctore sancti moniali Heidenheimensi, O. HoldER-EGGER éd., MGH SS, 15/1, Hanovre, 1887, p. 86-117. L'édition présente deux textes distincts : la Vita Willibaldi episcopi Eichstetensis, p. 86-106 (BHL n $\left.{ }^{\circ} 8931\right)$ et la Vita Wynnebaldi abbatis Heidenheimensis, p. 106-117, (BHL n 8996).

14. I. N. Wood, The Missionary Life..., op. cit., p. 57-99.

15. Alcuin, Vita Willibrordi, dans C. Veyrard-Cosme éd., L'CEuvre hagiographique en prose d'Alcuin. Vitae Willibrordi, Vedasti, Richarii, Florence, 2003, p. 32-74 (BHL n 8935). 
gée en $731{ }^{16}$. En revanche, l'ouvrage eut une influence déterminante sur la Vie de Willibrord. Bède s'inspira quant à lui du récit de vie composé vers 715 par un religieux de Ripon nommé Stéphane, pour rédiger les passages de son œuvre consacrés à Wilfrid, évêque d'York et voyageur, qui fut l'un des premiers insulaires connus à tenter d'évangéliser les Frisons ${ }^{17}$. En plus de ces relations intertextuelles, des relations d'amitié ou de parenté unissaient souvent les missionnaires entre eux ou les missionnaires à leurs hagiographes ${ }^{18}$.

On peut s'attendre à ce que les textes, qui se répondent les uns aux autres sur la question de la mission, partagent des références communes sur celle du voyage religieux. Tous les auteurs sont d'origine insulaire. Trois d'entre eux, Hugeburc, Willibald et Alcuin, vivaient sur le continent ; Stéphane avait eu l'occasion de visiter Rome ${ }^{19}$. Les « lieux communs » de ces récits peuvent être hérités de la tradition chrétienne, ne pas rendre compte de toute la singularité de chacun des voyages qu'ils décrivent, ils témoignent pourtant un peu de la manière dont les membres de ce petit groupe d'individus, saints et hagiographes, se racontaient, à eux-mêmes, leur expérience pérégrine.

\section{L'idéal de la peregrinatio}

\section{Désir d'exil et surenchère ascétique}

La pratique du voyage spirituel repose sur l'aspiration du fidèle à se défaire de ses attaches terrestres. Boniface, avant de faire voile vers la Frise, « commença [...] à éviter le commerce de ses parents et de ses proches (parentum adfinumque suorum consortia) et à désirer [partir vers] des lieux étrangers plutôt que [de rester dans] ceux des terres de l'héritage paternel. Après avoir mûrement considéré la question de quitter ses parents et sa patrie », il déclara ses intentions à l'abbé de Nursling ${ }^{20}$. Hugeburc évoque elle aussi le désir qu'éprouve Willibald, qui se rendra bientôt à Rome, de quitter « sa patrie, ses parents et ses proches » (patria et parentes atque propinqui) auxquels elle joint

16. I. N. Wood, The Missionary Life..., op. cit., p. 45 ; BÈDE, Historia Ecclesiastica Gentis Anglorum, dans B. Colgrave et R. A. B. Mynors éd., Bede's Ecclesiastical History of the English people, $3^{e}$ éd., Oxford, 2007 (désormais BèDE, HE).

17. D. P. KIRBy, « Bede, Eddius Stephanus and the Life of Wilfrid», English Historical Review, 98, 1983, p. 101-114.

18. I. N. Wood, The Missionary Life..., op. cit. Des liens personnels existaient entre Stéphane et Wilfrid, entre Wilfrid et Willibrord, entre Willibrord et Boniface, des liens familiaux entre Alcuin et Willibrord, ainsi qu'entre Hugeburc, les deux frères et Boniface.

19. StÉPhANe, Vita Wilfridi, ch. 49, p. 100 ; ch. 52, p. 108.

20. Willibald, Vita Bonifatii, chap. 4, p. 15, 1. 8-19. 
les « richesses temporelles de ce monde ${ }^{21} »$. Alcuin, à propos de l'exil d'Egbert et de Wigbert en Irlande, cite encore pour principale raison du voyage « l'amour de la patrie céleste ». Il résume ce dont les exilés souhaitaient se détacher dans la trilogie de « la maison, la patrie et la famille ${ }^{22} »$. Les associations parentes / adfines / paternae hereditatis terrae, patria / parentes / propinqui et domus / patria / cognatio, qui sont un topos de l'hagiographie voyageuse ${ }^{23}$, renvoient à deux passages des Écritures. L'un concerne la « Vocation d'Abraham », dans lequel Yahvé ordonne au descendant de Sem de quitter « ton pays, ta parenté et la maison de ton père (de terra tua et de cognatione tua et de domo patris tuis) pour le pays que je t'indiquerai ${ }^{24} \gg$. L'autre, extrait des Évangiles synoptiques, est connu sous le nom de « récompense promise au détachement »: dans l'Évangile selon Matthieu, Jésus déclare que « quiconque aura quitté maisons, frères, sœurs, pères, mères, enfants ou champs à cause de mon Nom, recevra le centuple et aura la vie éternelle en partage ${ }^{25} \gg$.

Vocationnel, le voyage est l'occasion d'inaugurer une relation directe entre le fidèle et Dieu. Le champ lexical du désir (optare, desiderare, cupere, amor peregrinationis, ardor...) est abondamment mobilisé dans les passages consacrés au départ des saints. Cela se double souvent d'allusions à la jeunesse des peregrini ${ }^{26}$. L'ardeur ressentie par le candidat pérégrin doit lui permettre de franchir les obstacles qui s'opposent à son départ et tout particulièrement d'obtenir le consentement de ses proches et de ses supérieurs. Parce que son aspiration s'accorde avec la volonté divine, Boniface parvient à convaincre son abbé de le laisser prendre la route ${ }^{27}$. Le projet d'Egbert de quitter l'Irlande pour le continent n'aboutit pas, mais il faut l'intervention directe de Dieu, sous la forme de songes et de tempêtes, pour le dissuader de prendre le large. D'ailleurs, à en juger par la suite du récit, l'erreur d'Egbert ne fut pas tant d'écouter son désir d'exil que de penser que son voyage devait le conduire ailleurs que sur l'île d'Iona ${ }^{28}$. Cette conception de la pérégrination n'est pas réservée au seul contexte hagiographique. Dans une lettre écrite à l'abbesse Bugga, qui lui a demandé conseil à propos d'un voyage à Rome, Boniface se déclare incapable

21. Hugeburc, Vita Willibaldi, chap. 2, p. 89, 1.31-33.

22. Alcuin, Vita Willibrordi, chap. 4, p .42.

23. Par exemple : JÉrôME, Ep. 108, «Epitaphum sanctae Paulae », § 6, dans Lettres, J. LABOURT éd., Paris, 1949, t. 5, p. 163.

24. Gn 12.1-2.

25. Mt 19.27-29; Mc 10.28-31; Lc 18.28-30. Voir aussi JérôME, Ep. 108, § 3.

26. Boniface est déjà assez âgé lorsqu'il part pour la Frise. On verra en revanche STÉPHANE, Vita Wilfridi, ch. 3, p. 8 ; Alcuin, Vita Willibrordi, ch. 4, p. 42 ; Hugeburc, Vita Wynnebaldi, ch. 2, p. 107 ; Hugeburc, Vita Willibaldi, ch. 3, p. 90.

27. Willibald, Vita Bonifatii, ch. 4, p. 15.

28. BÈDE, $H E, \mathrm{~V}, 9$, p. $474 s q$. 
de prescrire ou d'interdire le départ et ne peut que recommander à la religieuse de suivre les voix de son for intérieur ${ }^{29}$. La relation directe des peregrini avec la divinité leur permet de se soustraire à l'autorité de la hiérarchie ecclésiastique, dont l'approbation, indispensable selon la Règle ${ }^{30}$, devient un signe de la bienveillance divine dans la logique du récit. La justification du mouvement par le désir a des précédents dans la littérature chrétienne ${ }^{31}$. La volonté de Dieu s'exprime directement, matériellement, par l'inspiration, l' « anxiété » qui saisit les corps des voyageurs, par les nombreux augures qui font parler le monde.

L'exil spirituel est plus qu'un renoncement aux choses matérielles ; il en est une réactualisation, un effort pour rendre ce détachement plus concret. Il n'est pas peu paradoxal que le désir de partir saisisse si souvent des hommes et des femmes, qui, en entrant en religion, ont déjà renoncé aux affaires du siècle. Le même passage des Écritures ${ }^{32}$ que celui mobilisé dans la Vie de Wilfrid ou dans l'Histoire ecclésiastique pour justifier des voyages vers Rome est employé par l'auteur de la Vie de Boniface pour rendre compte de l'entrée du jeune oblat dans la communauté monastique d'Exeter ${ }^{33}$. Lorsqu'un peu plus tard le religieux décide de quitter son île natale, le thème du renoncement est répété sans que cela ne pose d'apparentes difficultés à l'auteur. Alors qu'il se trouvait déjà au monastère de Ripon, Willibrord «s'enflamma ardemment pour une vie plus austère (artioris vitae succensus) et fut saisi par un vif désir de voyager (peregrinationis amore instigatus $)^{34} »$. Dans un autre monastère insulaire, Willibald, d'après Hugeburc,

se demandait jour et nuit comment se mêler à la chaste compagnie des moines, et de quelle manière il pourrait à l'avenir prendre part à l'heureuse discipline de leur vie en commun. Il commença alors à se demander anxieusement comment cette idée pourrait être appliquée en réalité et s'il ne serait pas souhaitable d'abandonner et de mépriser toutes les choses transitoires de ce monde $[\ldots]$ en $\mathrm{s}$ 'aventurant dans des terres étrangères et en gagnant des contrées inconnues ${ }^{35}$.

29. Lettre 27 (Boniface), p. 48. Cf. aussi (quoique la lettre 27 n'en soit pas une réponse directe) Lettre 14 (Eangyth et Bugga), p. 25.

30. Sur l'importance de la sanction hiérarchique, voir par exemple les $4^{\mathrm{e}}$ et $13^{\mathrm{e}}$ canons du concile de Chalcédoine (451) ; le concile anglo-saxon d'Hertford (673), cité par Bède (HE, IV, 5, p. 350) ; la Règle de saint Benoît (Regula S. Benedicti, 61, H. RochaIs éd. et trad., La Règle de saint Benoit, Paris, 1980, rééd. 1997, désormais RSB). Sur l'usage de lettres de recommandations : Willibald, Vita Bonifatii, ch. 5, p. 19 et 21.

31. Ainsi dans l'Itinéraire d'Égérie: J. SOLER, Écritures du voyage : Héritages et inventions dans la littérature latine tardive, Paris, 2005, p. 376-379.

32. Mt 19.27-29.

33. Willibald, Vita Bonifatii, ch. 1, p. 7 ; Stéphane, Vita Wilfridi, ch. 4, p. 10 [Mt 19.29] ; BÈDE, $H E$, V, 19, p. 516 [Mc 10.29-30 ; Lc 18.29-30].

34. Alcuin, Vita Willibrordi, ch. 4, p. 42.

35. Hugeburc, Vita Willibaldi, ch. 2, p. 89. 
Dans ces deux exemples, la peregrinatio, qui permet d'accéder à une " vie plus austère » et même d'appliquer « en réalité » (in effectum) l'idéal monastique, apparaît non seulement comme le prolongement naturel de la vie en communauté mais est présentée comme lui étant supérieure. Cette quête renouvelée d'étrangement au monde rappelle l'érémitisme méditerranéen. Comme lui, elle est justifiée par des raisons diverses qui peuvent aller du désir d'ascèse (artior vita) à la quête de quiétude dans une cellule d'un monastère étranger ${ }^{36}$, en passant par la volonté d'ignorer la gloire temporelle offerte dans sa patrie d'origine ${ }^{37}$. Comme l'idéal ancien de la xénitéia, elle pousse parfois les peregrini à chercher un exil plus lointain lorsqu'ils ne sont plus satisfaits de celui dans lequel ils se trouvent ${ }^{38}$. Bède et Alcuin signalent discrètement qu'Egbert et Willibrord ont abandonné l'Irlande pour d'autres lieux d'exil ${ }^{39}$. Hugeburc en revanche n'hésite pas à renouveler le thème du renoncement au sujet du départ de Willibald, qui abandonne un monastère italien pour un voyage en Terre sainte ; celui-ci, précise-t-elle, fut aussi mené, quoiqu'en toute indépendance, « sous la règle de la vie monastique ${ }^{40}$ ».

\section{L'ailleurs métaphorique et la question des destinations}

On constate, lors de l'évocation du premier départ des peregrini, une certaine indifférence des hagiographes à l'égard de la destination des expéditions. Les motivations concrètes des voyageurs de même que les lieux choisis pour leurs exils ne sont souvent mentionnés que dans un second temps et l'accent porte bien davantage sur le désir de départ. L'auteur de la Vie de Boniface décrit la naissance du projet de quitter sa patrie dans l'esprit du saint homme sans préciser ses ambitions missionnaires ; il relate ensuite la démarche que le religieux entreprend pour obtenir le consentement de son abbé, le soutien dont il bénéficie de la part des autres moines, le trajet vers Ludenwich (Londres), et ne mentionne la destination finale que lorsqu'il rapporte l'arrivée du voyageur à Dorstet (Dorestad), en Frise ${ }^{41}$. Il s'agit là, à n'en pas douter, d'un procédé littéraire. L'hagiographe signale que le voyageur embarque aux côtés de marins qui s'apprêtent à rentrer chez eux ${ }^{42}$ : ils sont donc Frisons et il est peu probable que Boniface, qui a dû discuter les tarifs avec eux, ait manqué de le remarquer.

36. Lettre 27 (Boniface), p. 48.

37. Willibald, Vita Bonifatii, chap. 4, p. 15, 1. 8-10.

38. A. Guillaumont, « Le dépaysement... », loc. cit., p. 104.

39. Alcuin, Vita Willibrordi, ch. 5, p. 44 ; Bède, HE, V, 9, p. 474-476.

40. Hugeburc, Vita Willibaldi, ch. 4, p. 92.

41. Willibald, Vita Bonifatii, ch. 4, p. 15-16.

42. Ibid. 
Le plus souvent d'ailleurs, le but des pérégrinations est évoqué assez tôt. Il n'empêche que l'organisation du récit, les tournures de phrases témoignent que cette perception de ce que doit être une vraie peregrinatio est largement répandue. Alcuin nous dit que Willibrord souhaite mener une vie plus austère et voyager; c'est alors seulement qu'il annonce (et quia) que le jeune homme " apprit que les études scolaires avaient pris de la vigueur en Hibernie ${ }^{43}$ ». Lorsque Hugeburc raconte le départ de Willibald pour Rome, elle parle d'abord de son aspiration à «parcourir des terres étrangères » et ne fait allusion au tombeau de saint Pierre que lorsque le religieux essaie de convaincre son père de venir avec lui ${ }^{44}$. Bède est sans doute l'auteur qui se donne le moins volontiers à ce lieu commun de la littérature pérégrine. Il s'empresse de préciser les raisons objectives des nombreux départs vers l'Irlande : la poursuite de la vie monastique ou l'étude des Écritures ${ }^{45}$. Cette disposition le rend un peu obscur lorsqu'il confie qu'Egbert avait envisagé tout à la fois de christianiser les Germains ou, si cela n'était pas possible, de se rendre à Rome pour adorer la basilique des apôtres et des martyrs, sans expliquer quel point commun pouvaient avoir les deux projets ${ }^{46}$. Il n'ignorait pourtant pas le thème de l'indifférence à l'égard de la destination réelle des exils spirituels, qu'il mobilise, par exemple, à propos du voyage de l'Irlandais Fursa en Est-Anglie ${ }^{47}$.

\section{« Vivre » une métaphore}

Le voyage est l'occasion de vivre une métaphore dont on trouve l'origine dans les textes patristiques. Gerhart Ladner a montré qu'il était possible de distinguer deux types d'aliénations symboliques dans les sources du début du Moyen Âge, chacun bénéficiant d'un vocabulaire spécifique pour le désigner ${ }^{48}$. D'un côté, le monde terrestre et les tentations qu'il inspire sont présentés comme «étrangers » à l'ordre divin, et donc au chrétien qui doit s'en détacher. De l'autre, ce détachement, seconde aliénation, définit la situation du fidèle dans le siècle. Ces représentations informent la métaphore de l'homme voyageur. Après la Chute, la vie terrestre ne peut plus être qu'un exil pour le

43. Alcuin, Vita Willibrordi, ch. 4, p. 42.

44. Hugeburc, Vita Willibaldi, ch. 2, p. 89; ch. 3, p. 90.

45. BÈDE, HE, III, 27, p. 312 ; O. SZERWINIACK, « Les relations entre l'Irlande et l'Angleterre dans l'Histoire ecclésiastique de Bède le Vénérable », F. Bourgne et al. (dir.), Un espace colonial et ses avatars. Naissance d'identités nationales : Angleterre, France, Irlande ( $V^{e}-X V^{e}$ siècles), Paris, 2008, p. 19-39.

46. BÈDE, $H E, \mathrm{~V}, 9$, p. 474, 476.

47. Ibid., III, 18, p. 268.

48. G. Ladner, «Homo Viator : Medieval Ideas on Alienation and Order », Speculum, 42, 1967, p. 233-259. 
fidèle qui doit rejoindre sa vraie patrie, la patrie céleste. Le chrétien est un étranger, un voyageur (peregrinus ou viator), parce que le monde lui est étranger, extérieur (alienus). L'image de l'homo viator a précédé nos sources de plusieurs siècles. Ladner en constate la présence, par exemple, dans les écrits de Grégoire le Grand ${ }^{49}$. L'image a également été utilisée par Augustin pour rendre compte de la condition temporelle de l'assemblée des fidèles, la civitas Dei peregrinans ${ }^{50}$. On sait l'influence qu'eurent ces auteurs sur la tradition insulaire ${ }^{51}$. Bien sûr, le trope puise sa source dans les Écritures. En latin classique, la notion de peregrinatio n'a pas de connotation religieuse particulière. Elle désigne un voyage à travers le pays (per ager) et par extension un voyage à l'étranger ${ }^{52}$. Le Deutéronome prescrit au peuple d'Israël «d'aimer l'étranger (peregrinus) car au pays d'Égypte vous fûtes des immigrés (advenae) ${ }^{53} \gg$. Le voyageur, qui n'est ni un religieux ni même peut-être un croyant, doit être accueilli parce que le peuple élu se reconnaît en lui. Plus proche de nos sources, Benoît de Nursie recommande qu'une attention particulière soit donnée à l'accueil des «frères dans la foi et des étrangers » (domestici fidei et peregrini) ou des « pauvres et des étrangers » (pauperes et peregrini), parce qu'en eux on accueille davantage le Christ ${ }^{54}$.

Dans les textes de notre corpus, cet usage classique du vocabulaire pérégrin persiste. Stéphane parle de l'exil du prince Dagobert en Irlande comme d'une peregrinatio, emploi qu'il justifie immédiatement par une référence au peule d'Israël ${ }^{55}$. L'auteur, d'ailleurs, avait de bonnes raisons de donner une signification biblique à l'exil politique puisque le saint dont il écrivait l'histoire dut à plusieurs reprises fuir son évêché pour échapper à l'animosité de la noblesse northumbrienne. Le terme peregrinatio apparaît ailleurs, au discours indirect, dans la bouche d'un dignitaire laïque, pour qualifier l'exil très politique de Wilfrid dans les royaumes du sud de la Bretagne ${ }^{56}$. Bède s'inspire de la Règle lorsqu'il évoque la générosité d'Oswald envers « les pauvres et les

49. Par exemple, GréGoire, Moralia in Iob, VIII, 54, M. Adriaen éd., Turnhout (CCSL, 143), 1979, p. 454 ; cité dans G. LadNeR, « Homo Viator... », loc. cit., p. 235-236.

50. Voir G. Clark, « Pilgrims and Foreigners : Augustine on Travelling Home», dans L. ElLis et F. KIDNER éd., Travel, Communication and Geography in Late Antiquity : Sacred and Profane, Burlington, 2004, p. 149-158.

51. Sur Grégoire le Grand, $c f$. BèdE, $H E$, II, 1 p. 126, 128 ; Lettres 33, 54, 59, 60, 75, 78 (Boniface) ; D. Rollason, « Bede and Germany », Jarrow Lectures, 2001, p. 18-19. Sur Augustin, cf. Lettres 50, 59, 64, 104.

52. G. Constable, « Monachisme et pèlerinage... », p. 4 ; D. WebB, Pilgrims..., p. 7-8.

53. Dt 10.19 .

54. $R S B, 53.1-2$ et 53.15 .

55. StÉPHANE, Vita Wilfridi, ch. 23, p. 68.

56. Ibid., ch. 40 , p. 80. 
étrangers ${ }^{57} \gg$. Il utilise aussi la métaphore plus tardive du chrétien voyageur dans la copie d'une lettre qu'il attribue à Ceolfrid ${ }^{58}$. Il n'est pas rare non plus de trouver dans les sources le rapprochement entre la métaphore de l'homo viator et la situation réelle des exilés religieux. Alcuin, reprenant le thème de la « patrie céleste » qu'il a déjà évoqué à propos du voyage d'Egbert et Wigbert en Hibernie $^{59}$, dit de Willibrord, au moment de sa mort, qu'il « a quitté cette pérégrination pour rejoindre la patrie éternelle ${ }^{60} »$. L'hagiographe parle certes de la vie terrestre mais il rappelle aussi bien que le saint, dès son départ de Bretagne, s'acheminait vers cette destination spirituelle. Un peu de la même manière, Cuthbert écrit à quelques mots d'intervalle que Lull, un religieux anglo-saxon, se trouve en Germanie dans « une pérégrination si dangereuse, si cruelle et si entière pour l'amour de la patrie éternelle » et que Boniface, qui vient de mourir en martyr dans son exil continental, a atteint quant à lui « la tranquillité éternelle de la patrie céleste ${ }^{61} »$. En dernière analyse, les «voies inconnues de la pérégrination ${ }^{62} »$ ne mènent pas tant à Rome, à Jérusalem ou en Germanie qu'à la « vie éternelle » que promettent les Évangiles, ou mieux à la «patrie céleste » que Bède évoque si souvent à propos des voyages religieux ${ }^{63}$.

Les auteurs anglo-saxons connaissaient donc la signification primitive du terme peregrinus, celle d'étranger réel, tout autant que la signification religieuse qui lui est donnée dans les Textes. Dans leurs écrits, le vocabulaire pérégrin se rapporte, dans un sens classique encore actif, à l'exil et au déplacement physique comme à une activité laïque, séculière par sa forme. C'est à ce simple exil que les peregrini pro Domino se livrent d'abord. Les auteurs n'hésitent pas à utiliser les termes exul ou exulare pour qualifier leurs voyages ${ }^{64}$. Des expressions telles que «pour Dieu », «pour le Christ », ou le recours aux Écritures sont nécessaires pour distinguer cette forme de dévotion de tous les autres types de déplacements. Pour autant, même lorsque le vocabulaire pérégrin est, comme chez Stéphane, appliqué à des voyages laïcs, on peut s'attendre à ce qu'il renvoie à des représentations bibliques, au peuple d'Israël ou à l'exil terrestre du Christ.

C'est à la lumière de ce premier constat, qu'il faut nous intéresser à une caractéristique souvent remarquée des usages lexicaux de Bède ${ }^{65}$. Lorsque ses

57. BÈDE, $H E$, III, 6, p. 230.

58. Ibid., V, 21.

59. Alcuin, Vita Willibrordi, ch. 4, p. 42.

60. Ibid., p. 66.

61. Lettre 111 (Cuthbert), p. 239.

62. StÉPHANe, Vita Wilfridi, ch. 3, p. 8 : inattrita via gentis nostrae; HugEBurc, Vita Willibaldi, ch. 3, p. 90 : ignotae peregrinationis viae; patulas peregrinationis rurae.

63. BÈDE, HE, III, 13, p. 252 ; V, 9, p. 476 ; V, 10, p. 480.

64. Ibid., III, 4, p. 224 ; V, 10, p. 480 ; V, 19, p. 516.

65. Le même type d'usages se retrouve dans les textes de notre corpus, à l'exception du récit 
choix de vocabulaire ne sont pas dictés par la tradition, l'auteur réserve avec une constance singulière (seize emplois sur dix-neuf) le nom peregrinatio et ses dérivés à des déplacements assimilables à des voyages spirituels, dont les destinations se trouvent toujours outre-mer ${ }^{66}$. Dans dix de ces seize occurrences, des thèmes ascétiques sont mobilisés pour qualifier ces mêmes voyages ${ }^{67}$. Les six emplois restants se rapportent à des types de déplacements à propos desquels les auteurs sont susceptibles de mobiliser les mêmes motifs, mais qui restent proches de l'usage classique ${ }^{68}$. À l'inverse, Georges Tugène rappelle justement que Bède n'utilise jamais ce vocabulaire pour qualifier des exils politiques, pourtant nombreux dans son récit. Il suggère que l'auteur a été influencé dans ses emplois par une particularité de sa langue maternelle, dans laquelle la terminologie relative à l'exil séculier renvoie à l'idée de vengeance et de persécution, conceptions qui sont étrangères à la démarche des peregrini pro Domino. Tandis que le latin attire l'attention sur le résultat de l'exil, l'expatriation et l'acte de voyager, le vieil anglais insiste sur les raisons qui en sont à l' origine ${ }^{69}$. Ce problème de traduction a pu avoir d'autant plus d'impact sur les usages lexicaux de Bède que, depuis l'Antiquité tardive, une longue polémique sur la valeur du voyage religieux agite la tradition chrétienne, qui a, de son côté, tendu à définir plus précisément ce que devait être une vraie pérégrination contre toutes les autres formes de déplacement.

Bon nombre de textes visant à réguler le mouvement des moines et des clercs sont adoptés par les conciles de l'Église à partir du IV siècle ${ }^{70}$. L'objectif de ces canons était de protéger l'Église établie contre le désordre apporté par

de Stéphane. Cependant, les allusions à d'autres formes de voyages que des voyages religieux sont trop peu nombreuses pour que cela soit significatif. Nous nous intéressons donc surtout à l'Histoire ecclésiastique.

66. P. F. Jones, A Concordance to the Historia Ecclesiastica of Bede, Cambridge (Mass.), 1929, p. 388-389. On leur ajoutera le canon du concile de Hertford visant « les évêques et les clercs en voyage » $(H E, I V, 5$, p. 350) : cet usage répond à celui du chapitre 61 de la Règle, De monachis peregrinis, qualiter suscipiantur.

67. À propos de Willibrord en Irlande (BèDE, $H E$, III, 13, p. 252); de l'Irlandais Fursa en EstAnglie (HE, III, 19, p. 258) ; d'Egbert en Irlande (HE, III, 27, p. $312 ; H E$, IV, 3, p. 344 ; HE, V, 9 , p. 474) ; de Hild en Gaule (HE, IV, 23, p. 406, deux occurrences) ; de Withbert en Irlande (HE, V, 9, p. 478-480, deux occurrences).

68. Dans cinq cas sur six, l'adjectif peregrinus (souvent employé au discours indirect) désigne des étrangers bénéficiant de prérogatives ou soumis à certaines contraintes dans les communautés (religieuses, politiques, etc.) qui les accueillent : BèdE, HE, I, 23, p. 68 ; III, 8, p. 238 ; V, 10, p. 482 ; $\mathrm{V}, 19$, p. $520 ; \mathrm{V}, 19$, p. 520 . Le terme peregrinatio est aussi employé, dans un sens presqu'étymologique, à propos du voyage long et difficile des compagnons d'Augustin vers la Bretagne (HE, I, 23, p. 68).

69. Il s'agit du substantif wraecca et du verbe wracian: G. Tugène, L'Image de la nation anglaise dans l'Histoire Ecclésiastique de Bède le Vénérable, Strasbourg, 2001, p. 151 sq.

70. Voir les références données en n. 3 à 5 . 
les religieux errants ou isolés, dont il n'était pas toujours facile de s'assurer de l'orthodoxie. Ces efforts normatifs accompagnaient souvent une condamnation morale du voyage. Dans les typologies des moines produites entre la fin du IV et le milieu du vi ${ }^{e}$ siècle, les religieux sans supérieurs, « sarabaïtes » ou « gyrovagues », sont volontiers accusés de camoufler leur concupiscence sous les apparences d'une vie pieuse ${ }^{71}$. Une grande partie des griefs contre le voyage qui sont exprimés dans nos sources s'inscrit dans cette tradition. On trouve dans la correspondance de Boniface de nombreuses invectives contre les prêcheurs isolés qualifiés de "faux prêtres » ou de «faux évêques ${ }^{72}$ », voire de "gyrovagues », ces «moines tonsurés » qui « ont fui leur maître » et ne sont asservis qu'à leur propre volonté ${ }^{73}$. Sous l'influence de Boniface, les conciles de l'Église franque réaffirmèrent le principe d'obédience, la supériorité de la norme bénédictine et défendirent aux autorités locales de laisser entrer au service de l’Église des prêtres et des évêques inconnus sans le consentement du synode ${ }^{74}$. Au-delà des désordres éventuels qu'auraient pu causer des religieux étrangers dans les Églises locales, les auteurs s'interrogent sur l'intérêt du mouvement physique pour le progrès spirituel des voyageurs. Bède critique discrètement l'habitude que ses contemporains avaient prise de se rendre à Rome « à l'envi » (certatim), voyage qui, nous dit-il ailleurs, avait pu autrefois (eo tempore) être considéré comme « un acte de grand mérite ${ }^{75}$ ». L'attaque est profonde, quoique discrète, puisqu'elle met en cause l'utilité même des voyages vers la Ville sainte $^{76}$. Vers 747, Boniface demande à l'évêque Cuthbert de Canterbury d'empêcher les « jeunes filles et femmes voilées » de partir pour Rome afin de protéger leur vertu et de sauver l'honneur de l'Église des Angles ${ }^{77}$. Le mouvement physique peut, dans certaines conditions, conduire le fidèle à la perdition en le plongeant plus profondément dans les turpitudes de la vie séculière plutôt que de le mener à Dieu par le renoncement aux choses du monde. Cette critique fait écho à la longue tradition patristique qui tend à distinguer la pérégrination intérieure du mouvement matériel en considérant que l'exil peut servir la

71. M. DiETZ, Wandering Monks..., op. cit., p. 73-78 ; D. Caner, Wandering, Begging Monks..., op. cit., p. 7 sq.

72. Lettres 57, 60, 63, 83, 88.

73. Lettre 80 (Zacharie à Boniface), p. 175-176; parmi ceux-ci se trouvaient des voyageurs au long cours dont l'Irlandais Sampson, cité dans la même lettre (p. 177).

74. Lettre 56 (Carloman), p. 100.

75. BÈDE, $H E$, V, 7, p. 472 et $H E$, IV, 23, p. 408.

76. On verra aussi JÉRôMe, Ep. 58, § 2-5, t. 3, p. 75-80 et G. Constable, « Monachisme et pèlerinage... », loc. cit.,p. 15.

77. Lettre 78 (Boniface), p. 169 : Boniface ne fut pas aussi sévère envers les voyages des religieuses qu'il connaissait personnellement (Lettres 27, 62, 105). 
conversion de l'âme sans y suffire tout à fait ${ }^{78}$, ou en condamnant simplement le voyage comme inutile dans la quête de perfection morale ${ }^{79}$. Boniface ajoute à cela les périls particuliers qui guettent le religieux sur la route. Il n'est ni anodin, ni inédit dans la littérature chrétienne que l'expression de cette dernière préoccupation surgisse à propos de voyages féminins ${ }^{80}$.

Dans ce contexte de défiance envers le voyage, l'exploitation des thèmes pérégrins à des fins panégyriques est soumise à caution. $\mathrm{Si}$, même parmi les déplacements entrepris avec des intentions apparemment louables, certains peuvent être présentés comme désastreux sur le plan spirituel, il peut sembler raisonnable à Bède d'utiliser le vocabulaire pérégrin avec parcimonie et $a$ fortiori de ne pas l'appliquer aux déplacements qui ne s'inscrivent pas dans une démarche proprement spirituelle. On se souviendra aussi que l'auteur de l'Histoire ecclésiastique n'a pas les même raisons que l'hagiographe de Wilfrid de rappeler la signification religieuse du voyage en général. Il est plus libre de se faire l'écho de cette suspicion qui pèse sur le mouvement et se garde d'accorder une légitimité scripturaire inconditionnelle à toute forme d'errance. De leur côté, les hagiographes s'efforcent de réconcilier le déplacement physique des saints avec la pérégrination intérieure en donnant des preuves « matérielles » de l'adéquation du projet pérégrin au dessein divin. Ce travail d'écriture est rendu d'autant plus aisé que la métaphore est présentée comme activement vécue, que le voyage est censé être, par intention, un détachement du monde.

Cette tendance à différencier les voyages, et c'est là une particularité du corpus anglo-saxon ${ }^{81}$, semble avoir eu des conséquences sur la forme que prirent les exils spirituels volontaires ou sur le type de déplacement à propos desquels les auteurs acceptèrent de mobiliser ce motif hagiographique : pour Bède, le voyageur religieux, quel que soit le vocabulaire utilisé pour le désigner, ne peut trouver l'étranger qu'il recherche en Bretagne même ${ }^{82}$.

Les conditions que rencontrent les peregrini dans leurs différents lieux d'exil sont très hétérogènes et les hagiographes ne s'attachent pas particulière-

78. Ainsi Jérôme, Ep. 22, § 1, t. 1, p. 110-111 ; voir A. Gulllaumont, « Le dépaysement... », loc. cit., p. 93.

79. Augustin, Confessiones, 18.28 et 8.8.19, citées dans G. Clark, «Pilgrims and Foreigners... », loc. cit.

80. J. Tibbets Schulenburg, « Strict Active Enclosure and Its Effects on the Female Monastic Experience (ca. 500-1100) », dans J. A. Nichols et L. T. Shank éd., Medieval Religious Women : vol. 1, Distant Echoes, Kalamazoo, 1984, p. 51-86.

81. T. M. Charles-Edwards, « The Social Background to Irish peregrinatio », Celtica, 11, 1976, p. 43-46.

82. G. Tugène, op. cit., p. 175, n. 32. Les expressions relatives à l'exil religieux qui ne mobilisent pas le vocabulaire pérégrin se trouvent dans BèDE, HE, III, 4, p. 224 ; III, 27, p. 312 ; III, 27, p. $314 ; \mathrm{V}, 10$, p. $480 ; \mathrm{V}, 19$, p. 516. 
ment à dégager ce qui, dans l'outre-mer en général, permet aux voyageurs de trouver l'exil du monde matériel qu'ils se sont promis. Il existe certainement des espaces symboliques précis, « hors du monde », qui répondent à cette quête de dépaysement radical. Boniface ou Willibrord errent dans des contrées païennes ou semi-païennes, volontiers qualifiées de «barbares ». Willibald découvre dans le bassin méditerranéen des lieux qui, quoique terrestres, sont l'incarnation réelle d'un « ailleurs » textuel, celui de la Bible ou des auteurs anciens ${ }^{83}$. En revanche, les indications relatives aux difficultés de la vie ultramarine considérée dans sa globalité sont rares. Le problème de la communication linguistique n'apparaît par exemple que de façon marginale ${ }^{84}$. Il est également difficile d'identifier dans les sources un sentiment laïque et spontané de la patrie, antérieur à l'exil ascétique, tourné vers l'île de Bretagne tout entière. Comme le souligne Georges Tugène, si un sentiment de ce type existait en Bretagne, il serait davantage orienté vers les petites gentes des royaumes anglo-saxons que vers la gens Anglorum dans son ensemble ${ }^{85}$. Qui plus est, la Bretagne de Bède est divisée en différents peuples parlant leur propre langue, et il faut un certain effort d'abstraction à l'historien de l'Église anglaise pour établir le lien privilégié, de nature religieuse, qui unit la gens Anglorum à l'île dont elle n'occupe qu'une partie $^{86}$. Il existe cependant un milieu bien réel, commun à tous les voyages religieux, où la métaphore de l'homme étranger, soumis à la puissance d'une volonté qui le dépasse, peut s'actualiser sans contraintes. Cet espace déterritorialisé, ce «non-lieu » où peut s'incarner le voyage idéel, c'est la mer.

\section{La pérégrination et la mer}

Si la qualité informative des passages qui leur sont consacrés est oblitérée par les stéréotypes ${ }^{87}$, tous nos textes comportent des descriptions de traversées maritimes. Ces sources se sont montrées suffisamment précises pour identifier des lignes commerciales, localiser des infrastructures portuaires, glaner des indices relatifs aux techniques de navigation ou à la composition des

83. Sur ce thème, voir aussi la belle discussion de J. Soler, op. cit., p. 369-396.

84. Bède, HE, I, 23, p. 69 et III, 7, p. 235 ; Willibald, Vita Bonifatii, ch. 6, p. 28 ; M. Banniard, «Credo et langage : les missions de saint Boniface », dans A. Dierkens et J.-M. SANSTERre éd., Voyages et voyageurs..., op. cit., p. 165-187.

85. G. TuGÈnE, op. cit., p. 91-94, p. 133-134, p. 147-171.

86. Ibid., p. 91-94, p. 133-134, p. 147-171; P. Wormald, « Bede, the Bretwaldas and the Origins of the gens Anglorum » [1983], rééd. dans ID., The Times of Bede : Studies in Early English Christian Society and its Historian, Oxford, 2006, p. 106-134.

87. S. Matthews, The Road to Rome: Travel and Travellers between England and Italy in the Anglo-Saxon Centuries, Oxford, 2007, p. 36 ; M. МсСовміск, Origins of the European Economy, Cambridge, 2001, p. 402-403. 
équipages ${ }^{88}$. Les auteurs, dont plusieurs étaient exilés sur le continent, connaissaient le milieu marin. Ils étaient cependant plus familiers des routes terrestres que des voies navigables. Le recours aux stéréotypes, les allusions « gratuites », l'attrait pour le détail véridique témoignent de leur intérêt pour des traversées qui, bien qu'elles conservassent à leurs yeux un caractère exceptionnel, n'en étaient que plus significatives.

"Les voies inconnues de la mer » : de la traversée maritime au port du salut

Les traversées sont souvent l'occasion de développements lyriques. Dans une succession remarquable d'allitérations (oceanus, cerulea lympharum liquida, tumida equorum timida, congelida gurgitum, garrula fluctuum, spumosa syrtium salsa, plus loin sont évoqués les spumosae undarum equorae et periculosae ponti procellae), Hugeburc mobilise tout un lexique nautique pour évoquer le voyage de Wynnebald vers Rouen ${ }^{89}$. La mer glisse sous la plume de l'hagiographe, les noms communs sont multipliés sans parvenir à cerner l'élément marin. L'évocation fait appel aux sens plus qu'à l'intellect : l'océan est décrit par la couleur des flots (cerulae liquida), son immensité (pontes), le bruit (gurgites, garrula fluctuum; les allitérations) et même par son odeur et la sensation tactile qu'il produit peut-être sur le visage des voyageurs (spumosa syrtium salsa : «l'écume salée des écueils »).

Tous les hagiographes ne déploient pas les mêmes efforts pour décrire les voyages sur la mer, mais ils sont régulièrement l'occasion de montrer quelque talent littéraire. Willibald utilise une polyptote (celocis celeriter marginem scandens) pour souligner l'empressement avec lequel Boniface embarque pour un voyage longtemps différé. Le trope est bientôt suivi de l'allusion aux « immenses voiles » (immensa carbasa) et à l'agitation des marins (trepudientes nautae ${ }^{90}$. La rumeur de l'équipage attire aussi l'attention de Hugeburc dans la Vie de Willibald (remigiis crepitantis, classis clamantibus) ${ }^{91}$. Dans la correspondance de Boniface ou dans l'Histoire ecclésiastique de Bède, une terminologie héroïque (equorei campi, pelagus...) est souvent employée, en plus des noms communs mare et oceanus, pour désigner le grand large ${ }^{92}$. Le thème le plus susceptible de faire concurrence à celui du voyage en mer est sans doute le passage des cols monta-

88. S. LebeCQ, «Quentovic : un état de la question », dans H.-J. HäBler et C. LorRen éd., Studien zur Sachsenforschung, Band 8, 39, Hildesheim, 1993, p. 73-82 ; S. LEBECQ, Marchands et navigateurs frisons du haut Moyen Âge, vol. 1 : Essai, Lille, 1983, p. 165 sq., p. 178-179, p. 185-215.

89. Hugeburc, Vita Wynnebaldi, ch. 2, p. 107.

90. Willibald, Vita Bonifatii, ch. 5, p. 20.

91. Hugeburc, Vita Willibaldi, ch. 3, p. 91.

92. Lettres 14, 124 ; BÈDE, HE, III, 15, p. 260 ; IV, 16, p. 384 ; V, 1, p. 454. 
gneux ${ }^{93}$. Mais il faut alors se contenter d'allusions très brèves, qui, à la différence de celles relatives aux traversées maritimes, ne permettent pas de retracer l'itinéraire des peregrini ni n'éclairent les conditions matérielles des ascensions.

L'expérience d'un voyage en mer était éprouvante au début du Moyen Âge. Les passagers embarquaient à bord de bateaux de petite taille, aux voiles rudimentaires ${ }^{94}$. Les récits de tempêtes ${ }^{95}$ sont nombreux dans l'Histoire ecclésiastique. Celles-ci peuvent être suffisamment violentes pour briser les bateaux au mouillage ${ }^{96}$. Elles surprennent aussi les hommes au large, même lors de trajets assez courts comme celui de l'île de Farne à celle de Lindisfarne ${ }^{97}$. Les intempéries, les courants côtiers n'étaient pas faciles à appréhender et le risque était réel. La tempête, cependant, est toujours dotée d'un sens hautement symbolique. Ici, Aidan et Cuthbert apaisent les éléments déchaînés et démontrent leur sainteté. Là, Dieu détruit les bateaux d'Egbert pour le décourager de quitter l'Irlande. Ailleurs, ce sont les démons qui agitent les flots pour que de saints hommes n'arrivent pas en Bretagne ${ }^{98}$. Même lorsque l'issue du voyage est favorable, la menace de la mer reste très présente dans les sources hagiographiques. Parvenus à Rome sains et saufs, Wynnebald et Willibald remercient Dieu de les avoir protégés « des grands dangers de la mer et des diverses turpitudes liées à la pérégrination à l'étranger ${ }^{99}$ ». Les aléas des traversées maritimes méritent une mention particulière, distincte de celle des périls terrestres. Au moment de la traversée, l'hagiographe évoquait «l'effroi inspiré par les flots» et « les dangereuses charges de la haute mer ${ }^{100}$ ». Le thème des « vents favorables » et du « voyage prospère » répond aux craintes engendrées par un élément incontrôlable sur lequel Dieu seul peut faire valoir son emprise ${ }^{101}$. Wilfrid se rend en Frise poussé par un doux zéphyr d'Ouest qui souffle « selon son désir ${ }^{102}$ ». Ailleurs, lui et ses compagnons, « embarquant avec de nombreuses bénédictions et l'aide des reliques des saints, le vent soufflant selon le désir des marins, parvinrent sans encombre à un port abrité de leur pays ${ }^{103}{ }^{»}$.

93. Hugeburc, Vita Willibaldi, ch. 3, p. 91 ; Willibald, Vita Bonifatii, ch. 5, p. 20 ; StéPhane, Vita Wilfridi, ch. 55, p. 120.

94. S. LeBeCQ, Marchands et navigateurs..., vol.1, p. 187-189 ; M. MCCormick, Origins..., p. 403-404, p. 407-417, p. 428-429.

95. On se souviendra des modèles antiques : E. DE SAINT-DenIs, Le Rôle de la mer dans la poésie latine, Lyon, 1935.

96. BÈDE, HE, I, 2, p. 20 ; V, 9 p. 478.

97. Ibid., V, 1, p. 454 ; III, 15, p. 260.

98. Ibid., III, 15, p. 260 ; V, 1, p. 454 ; V, 9, p. 478 ; I, 17, p. 54.

99. Hugeburc, Vita Willibaldi, ch. 3, p. 91.

100. Ibid., ch. 3, p.91.

101. Ainsi Willibald, Vita Bonifatii, ch. 5, p. 20 : pleno vento, prosperoque cursu; cf. S. LEBECQ, Marchands et navigateurs..., op. cit., vol. 1, p.165 sq., p. 185-215.

102. StÉPHANE, Vita Wilfridi, ch. 26, p. 52.

103. Ibid., ch. 7, p. 14. 
Milieu étranger où la volonté divine s'exprime avec force, la mer est un lieu propice au voyage religieux. Lorsque Boniface embarque pour Quentovic, son hagiographe nous dit qu'il « commença à arpenter les voies inconnues de la mer » (ignotae maris viae) ${ }^{104}$. Cette expression fait écho à une autre, apparue quelques phrases plus tôt quand l'auteur évoquait « les longues voies de la pérégrination » (longae peregrinationis viae) ${ }^{105}$. De même, Willibald conçoit le désir de partir en même temps que celui de prendre le large :

Il souhaitait s'aventurer sur les voies inconnues de la pérégrination (ignotae peregrinationis viae), il voulait arpenter et voir des terres étrangères et avait décidé de parcourir les eaux très sauvages de la mer (immanissimae maris aequorae) ${ }^{106}$.

Mais le rapprochement entre voyage sur la mer et exil religieux est encore plus évident sous la forme métaphorique. Dans une série de lettres écrites au milieu des années 730 , toutes adressées à des correspondants insulaires, Boniface use avec une constance remarquable d'une image qui le met en scène lui, l'envoyé de l'Église, ballotté par les tempêtes des mers germaniques ${ }^{107}$. À cette époque, le religieux réformateur est confronté à la résistance de l'élite locale ${ }^{108}$. Les sujets qu'il aborde dans ses missives ont peu de rapport avec la navigation ; elles concernent le droit du mariage, le rite du baptême ou l'ingratitude du travail évangélique. Pour autant, l'exul Germanicus ${ }^{109}$ demande à ses interlocuteurs de prier pour lui afin que, « puisque la mer Germanique est dangereuse pour ceux qui naviguent, il puisse arriver, grâce à leur prière et sous la gouverne de Dieu, jusqu'au rivage de la paix éternelle sans tache et sans préjudice pour son âme ${ }^{110} »$. Il compare aussi sa peregrinatio (sa vie ou son exil ?) à un voyage en mer lorsqu'il réclame des prières

pour que le Seigneur miséricordieux, qui est cause de notre pérégrination, protège et gouverne de sa main notre navire fragile, afin qu'il ne soit pas englouti par les flots des tempêtes germaniques, et parvienne indemne au rivage tranquille de la Jérusalem céleste ${ }^{111}$.

104. Willibald, Vita Bonifatii, ch. 5, p. 20.

105. Ibid.

106. Hugeburc, Vita Willibaldi, ch. 3, p. 90.

107. Lettres 30 , p. 54 ; 31, p. 55 ; 32, p. 55 ; 33, p. $56-57$; 34, p. $58-59$; 38, p. 63.

108. Lettres 28, 43 ; T. F. X. Noble, dans E. EMERTon éd., The Letters of saint Boniface, New York, 2000, p. XIV-XX.

109. Lettre 30 (Boniface), p. 54.

110. Lettre 32 (Boniface), p. 55 : Germanicum mare periculosum est ; cf. aussi Lettre 34, p. 58 : Germanici maris tempestates; Lettre 33, p. 57 : varii Germanicarum gentium tempestatum fluctes.

111. Lettre 38 (Boniface), p. 63. 
Boniface n'ignorait pas que son exil réel dans les terres germaniques pouvait être considéré comme une peregrinatio ${ }^{112}$, ni ce que cette notion avait de métaphorique. La comparaison de la vie humaine à une traversée maritime a des antécédents dans sa correspondance. Dès 716, Boniface, alors en Frise, avertissait un correspondant insulaire que l'étude des Écritures était le plus sûr moyen de permettre au «navire de notre âme » d'échapper au «naufrage » et d'arriver « au rivage du paradis et à la joie éternelle des anges ${ }^{113}{ }$. L'image du navire de l'Église gouverné par son évêque apparaît à la fois sous la plume de Boniface et sous celle de Stéphane de Ripon ${ }^{114}$. Cette rhétorique nautique a été employée depuis l'Antiquité pour décrire la vie de l'homme, mais aussi celle de la Cité, de l'État et de l'Église ${ }^{115}$. D'ailleurs, les auteurs empruntent parfois explicitement leurs images aux Pères ${ }^{116}$. Plus que l'ancienneté du trope, ce qui compte ici est la fréquence avec laquelle les correspondants en font usage ; la manière aussi dont ils le vivifient, inventant pour leur propos l'idée de « mers germaniques »; l'empressement avec lequel, finalement, ils emboîtent les métaphores maritimes et voyageuses.

\section{Le sentiment d'insularité et la découverte de la patrie}

Dans une sorte de mouvement de retour, les textes et les lettres des exilés participent à forger l'idée de l'île-patrie. L'insularité est exprimée, surtout dans les correspondances, sous la forme d'un sentiment, celui de la séparation, toujours plutôt maritime que terrestre. Il est beaucoup question, bien sûr, des courriers et objets venus « d'outre-mer » (transmarinus, ultramarinus) adressés par les exilés religieux, parfois bien retirés en arrière des terres, à leurs correspondants restés sur l'île ${ }^{117}$. Empruntant à Jérôme, la religieuse Egburga exprime à Boniface son impatience de le revoir, en mobilisant abondamment des motifs aquatiques ${ }^{118}$. Pour les exilés, la séparation est aussi celle de la « patrie insu-

112. Lettre 14 (Eangyth à Boniface), p. 24 ; Lettre 27 (Boniface), p. 48 ; Lettre 94 (Boniface), p. 214 ; Lettre 111 (Cuthbert à Lull), p. 239.

113. Lettre 9 (Boniface), p. 6. Voir aussi, Lettre 14 (Eangyth à Boniface), p. 22 ; Lettre 49 (Lull à Cuniburg), p. 79.

114. Lettre 78 (Boniface), p. 164 ; STÉPHANE, Vita Wilfridi, ch. 21, p. 44. Stéphane évoque aussi les relations entre Wilfrid et le roi Aldfrith en termes maritimes : Vita Wilfridi, ch. 55, p. 90.

115. Voir C. Bonner, « Desired, Haven », Harvard Theological Review, 34/1, janvier 1941, p. $49-57$.

116. Lettre 78 (Boniface), p. 164-165 ; Julien Pomère, De vita contemplativa, I, 16, dans $P L$, vol. 59, col. 431C-432A.

117. Lettre 114 (Cinehard de Winchester), p. 247 ; Lettre 14 (Eangyth), p. 21 ; Lettre 94 (Boniface).

118. Lettre 13, p. 20 ; JÉRÔME, Ep. 3, § 2, t. 1, p. 12. 
laire ${ }^{119} »$. Le point de vue adopté est alors celui, rétrospectif, que portent les voyageurs sur leur terre natale. La diversité ethnique et la segmentation politique de la Bretagne y perdent toute pertinence. L'image peut s'accompagner de développements lyriques laissant une large place au milieu marin. Lull évoque son départ lors d'un voyage vers Rome avec un plaisir littéraire certain :

Après que, mû par l'appel - que je crois salutaire - de la miséricorde divine, j'eus quitté dans la hâte les illustres royaumes des terres britanniques, laissant derrière moi l'île très féconde de ma patrie natale, que les eaux sombres de la mer écumante, qui se heurtent contre les côtes rocheuses, entourent de tous côtés $[\ldots]^{120}$.

Un autre mode d'expression, géographique cette fois, de l'idée que la gens Anglorum est un peuple insulaire prend forme lorsque, confrontés à un regard étranger, les auteurs sont amenés à définir la place qu'occupe leur île dans un monde organisé autour de la Méditerranée. D'après Hugeburc, le « roi des Sarrasins » demande d'où viennent Willibald et ses compagnons, alors emprisonnés à Émèse. Leurs défenseurs répondent qu'ils sont originaires « des contrées occidentales, où le soleil se couche, nous ne savons rien de leur pays si ce n'est qu'au-delà il n'y a que de l'eau ${ }^{121} »$. Bède prête à Wilfrid un long discours sur la date de Pâques. Le premier argument du défenseur des pratiques romaines, fraîchement revenu d'un voyage sur le continent, est de rappeler que Pâques est célébrée par tous les peuples chrétiens à la même date. Par tous, ditil, « sauf [...] les Pictes et les Bretons, qui, depuis les deux îles de l'océan les plus éloignées, et même pas dans leur totalité, s'opposent à toute la terre avec une vigueur insensée ${ }^{122}$ ». Ce motif de la « lointaine Bretagne », hérité de la tradition continentale, se trouve également chez Gildas, dans l'œuvre d'Aldhelm, ou dans la lettre déjà citée de Cuthbert ${ }^{123}$.

Le thème de l'insularité se dote finalement de significations religieuses plus profondes ${ }^{124}$. Les premiers mots de la Vie de Willibrord lui sont consacrés

119. BÈDE, $H E$, III, 19, p. 274 ; III, 27, p. 312.

120. Lettre 98 (Lull), p. 219.

121. Hugeburc, Vita Willibaldi, ch. 4, p. 95 ; voir aussi StÉPhAne, Vita Wilfridi, ch. 51, p. 104 : de ultima terrarum parte.

122. BÈDE, HE, III, 25, p. 300.

123. Aldhelm, Epistulae, dans Aldhelmi Opera, MGH AA, t. XV, p. $492-493$; Lettre 11 (Cuthbert), p. 240, 1.1-2 ; voir S. SoBECKI, « Britain at the Last Cliff of Ocean », dans ID., The Sea and Medieval English Literature, Cambridge, 2008, p. 72-91 ; A. GAUTIER, «"Regards ethnographiques sur le rebord du monde". Les modèles alimentaires méditerranéens dans l'Angleterre du haut Moyen Âge ", dans J. Leclant, A. Vauchez et M. Sartre éd., Pratiques et discours alimentaires en Méditerranée de l'Antiquité à la Renaissance, Paris, 2008 (Cahiers de la Villa Kérylos, n 19), p. 293-315, sp. p. 293-295.

124. La riche littérature du monastère insulaire de Lérins a contribué à cette évolution en développant depuis le $v^{\mathrm{e}}$ siècle le thème de «l'île sainte ». 
(fuit in Brittannia insula...). Il réapparaît presque aussitôt lors de la description des « hauteurs qu'entourent l'Océan et la Humber ${ }^{125}$ » sur lesquelles Wilgils, le père de Willibrord, se retire pour mener une vie d'ermite. L'analepse crée un antécédent de sainteté au personnage principal du récit. Comme le remarque Christiane Veyrard-Cosme, l'exil insulaire, qui permet d'établir une relation plus intime avec Dieu, et le thème de l'eau, source régénérée de la vie, contribuent à la mise en scène d'un «état pré-lapsaire » duquel le missionnaire tirerait son origine ${ }^{126}$. Le promontoire isolé de Spurn Point est le point de départ du récit de la vie d'un saint qui sera amené à parcourir le monde. Il est aussi le lien qui lie l'auteur de la Vie à son dédicataire ${ }^{127}$. Tous deux sont issus de la même lignée, tous deux vivent sur le continent. L'un a hérité de la cellula fondée par le père de Willibrord, et l'autre se trouve à la tête du monastère d'Echternach, fondé en Austrasie par le saint lui-même. L'insularité est hautement symbolique, elle permet d'évoquer une «patrie » devenue complètement métaphorique et dont la qualité physique essentielle - de Spurn Point à la Bretagne - est d'être une île. Alcuin a sans doute été influencé par Bède, mais il faut attendre d'autres auteurs, continentaux, pour que l'histoire de toute la gens Anglorum et de « son île ${ }^{128} \gg$ soit absorbée par l'hagiographie missionnaire. L'auteur anonyme de la Vie de Lebuin, un texte composé entre la seconde moitié du $\mathrm{IX}^{\mathrm{e}}$ et le début du $\mathrm{X}^{\mathrm{e}}$ siècle, qui puise son inspiration dans les traditions des deux « familles ${ }^{129}$ », ouvre son récit en présentant brièvement les origines des Anglo-Saxons :

La patrie des Angles, convertie à la foi chrétienne par le zèle pieux du bienheureux pape Grégoire, fit toujours preuve d'une très grande piété. [...] Beaucoup choisirent de s'exiler pour le Seigneur, de sorte que, par le labeur de la pérégrination, ils purent soit s'affranchir de leurs propres péchés soit soutenir les païens ou les chrétiens dans leur éducation ${ }^{130}$.

Loup de Ferrières, peut-être en $836^{131}$, entame le récit de la Vie de Wigbert, un disciple de Boniface, en rappelant l'origine continentale des « habitants de la Bretagne qu'on appelle les Anglo-Saxons » et en retraçant rapidement l'histoire des migrations ${ }^{132}$. Dans les deux cas, les destins individuels des

125. Alcuin, Vita Willibrordi, ch. 1, p. 38-39 (trad. C. Veyrard-Cosme).

126. C. Veyrard-Cosme, L'Euvre hagiographique..., op. cit., p. 256.

127. I. N. Wood, The Missionary Life..., p. 80-81.

128. BÈDE, HE, V, 24, p. 570 : historia ecclesiastica nostrae insulae ac gentis ; cf. G. Tugène, L'Image de la nation..., op. cit., p. 166.

129. I. N. Wood, The Missionary Life..., op. cit., p. 115-117.

130. Vita Lebuini Antiqua, A. HofmeIster éd., MGH SS, t. XXX-2, p. 791 (BHL n 4810b).

131. I. N. Wood, The Missionary Life..., op. cit., p. 66.

132. Loup de Ferrières, Vita Wigberti abbatis Friteslariensis, O. Holder-EgGer éd., MGH SS, t. XV-1, p. 39, (BHL n $\left.{ }^{\circ} 8879\right)$. 
missionnaires sont donc réintroduits dans l'histoire collective du peuple des Angles. Si l'idée de l'insularité demeure à travers l'évocation de la Bretagne, c'est, après l'Histoire ecclésiastique dont ces auteurs s'inspirent, en référence à une certaine « géohistoire ». Celle-ci raconte comment, en s'emparant d'abord d'une ancienne province ecclésiastique, puis en se convertissant au catholicisme romain et en s'efforçant de le répandre dans l'île et sur le continent, le peuple des Angles se fit une place, " insulaire », dans l'histoire universelle et « continentale $»$ du christianisme ${ }^{133}$.

\section{Conclusion}

Deux tendances interprétatives se côtoient donc dans les textes pour rendre compte du mouvement pérégrin. L'une puise ses références dans les sources patristiques et leurs métaphores voyageuses. Son propos est de justifier le désir de départ et de donner à cet acte une valeur exemplaire : le voyage résume la place qu'occupe l'homme dans le monde, l'application littérale de la métaphore y est figurée comme un acte de soumission à la volonté divine. Elle appelle immédiatement la métaphore maritime. L'autre efface en partie cette relation directe des hommes à Dieu et explique les destins individuels par celui des nations. Elle suppose un certain « recul historique » et le constat préalable de la répétition de l'expérience missionnaire. Elle s'appuie sur l'idée que la Providence décide du devenir des peuples et détourne pour son usage des schémas développés par les auteurs chrétiens du continent et par les historiens et géographes de l'Antiquité grecque et romaine ${ }^{134}$. Elle ranime, en particulier, l'idée de la «Bretagne », en s'appuyant sur la notion, très symbolique et commune aux deux tendances, de l'île-patrie. Il se peut que le succès que connut cette interprétation du phénomène pérégrin dans les textes hagiographiques du début du $\mathrm{IX}^{\mathrm{e}}$ siècle soit lié à la méfiance croissante que l'on éprouvait dans l'Europe carolingienne envers l'errance religieuse ; à moins que cette explication « historique » n'ait simplement été plus familière et accessible aux auditoires continentaux auxquels les récits étaient adressés ${ }^{135}$. Les auteurs anglo-saxons

133. P. Worlmald, «Bede, the bretwaldas... », loc. cit.; D. Rollason, «Bede and Germany... », loc. cit. ; G. TugÈne, L'Image de la nation..., op. cit.

134. Cf. à ce sujet M. COUMERT, Origine des peuples : Les récits du haut Moyen Âge occidental (550-850), Paris, 2007, p. 403-439.

135. Dans les Vies de Lebuin, Leoba et Wigbert, l'exil est davantage « subi » que dans les textes du $\mathrm{VIII}^{\mathrm{e}}$ siècle puisque Leoba et Wigbert rejoignent Boniface à sa demande pressante (RoDOLPHE DE Fulda, Vita Leobae, éd. G. WaItz., MGH SS, XV-1, § 10, p. 125, BHL n 4845 ; Loup DE FERRIÈres, Vita Wigberti, § 4, p. 39) et que Lebuin obéit directement à Dieu (sans allusion au désir du saint) qui lui demande « lui-même » (Salvator ipse) de partir pour christianiser les païens (Vita Lebuini Antiqua, § 2, p. 791, 1. 30-34). Dans la Vie de Willehad, comme dans les textes précédem- 
du VIII ${ }^{\mathrm{e}}$ siècle, les missionnaires et leurs correspondants, eurent plus d'audace pour développer le thème du désir radical d'exil, et celui, associé, de la traversée maritime.

À l'effacement du thème du voyage dans les Vies des missionnaires anglo-saxons rédigées au $\mathrm{IX}^{\mathrm{e}}$ siècle sur le continent répond peut-être une évolution diamétralement opposée dans la littérature religieuse insulaire. Kathleen Hughes a montré comment, en Irlande, l'aspiration à l'exil ascétique, sous la pression de critiques domestiques à l'encontre du voyage religieux et en raison de la méfiance croissante que rencontraient les peregrini dans l'espace carolingien, dut se trouver de nouvelles formes d'expressions au tournant des $\mathrm{VIII}^{\mathrm{e}}$ et IX $\mathrm{X}^{\mathrm{e}}$ siècles. Parmi celles-ci, Hughes relève l'essor de récits de voyages chrétiens fictionnels accordant une place considérable à l'errance sur la mer ${ }^{136}$. Le poème religieux connu sous le nom de Seafarer pourrait témoigner d'une transformation similaire dans la littérature anglo-saxonne. Ce texte en vieil anglais, composé dans le courant du IX ${ }^{\mathrm{e}}$ siècle, présente les états d'âme d'un voyageur qui relate les peines de la navigation avant de présenter les avantages spirituels de la vie d'exilé. Dorothy Whitelock a plaidé en faveur d'une interprétation littérale du poème qui se réfère, pour elle, à l'exil ascétique d'un peregrinus insulaire ${ }^{137}$. Sebastian Sobecki a relevé une contradiction apparente dans cette lecture de l'œuvre ${ }^{138}$. Il remarque que, si le poète envisage effectivement le voyage en mer comme un moyen de se prémunir des séductions du monde matériel, sa quête de côtes étrangères, où les mêmes tentations ne manqueront pas de se présenter à lui, est quelque peu paradoxale. Il note également que l'on ne connaît pas d'exemple de voyageur anglo-saxon qui se soit adonné, comme certains Irlandais, à un exil sur les eaux plutôt qu'à un voyage dont la destination se trouvait au-delà de la mer.

Ces remarques sont justes, mais elles nous semblent oublieuses de la dimension métaphorique de la pratique-même de la peregrinatio pro Deo. Un homme tel que Boniface rechercha réellement un exil continental dans les terres germaniques tout en présentant ses pérégrinations comme une équipée maritime. Si l'auteur du Seafarer se référait effectivement à l'idéal de l'exil ascétique, il est compréhensible qu'il ait insisté davantage sur le thème du voyage en mer que sur celui, vivement contesté, de l'errance dans les terres. Ce faisant, il

ment mentionnés, la vocation évangélique l'emporte sur le thème de l'exil (Vita Willehadi, §1, éd. G. H. Pertz, $M G H$ SS, II, p. 380, BHL n 8898). Cf. G. Constable, « Monachisme et pèlerinage... », loc. cit., p. 10, n. 1 .

136. K. Hughes, « The Changing Theory...», op. cit., p. 148-151.

137. D. Whiтelock, «The Interpretation of The Seafarer », dans C. Fox et B. Dickins éd., The Early Cultures of North-West Europe, Cambridge, 1950, p. 259-274.

138. S. SoвескI, «The Interpretation of The Seafarer : A Re-examination of the Pilgrimage Theory », Neophilologus, 92, 2008, p. 127-139. 
offrait à son audience une mise en scène stylisée du voyage religieux, mais qui ne trahissait pas pour autant l'aspiration réelle des peregrini à vivre concrètement la métaphore de l'homo viator ${ }^{139}$.

AUTRES TEXTES INFLUENTS

\section{FAMILLE "BONIFATIENNE"}

$$
\begin{gathered}
\text { STEPHANE DE RIPON } \\
\text { Vita Wilfridi } \\
\text { Ripon } \\
710-715 / 731-734
\end{gathered}
$$

BONIFACE

(Wessex 675- Dokkum 754)

Correspondance

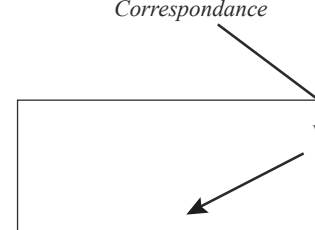

HUGEBURC

Vitae Willibaldi (après 763-768)

et Wynnebaldi (avant 786)

Heidenheim

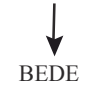

Historia Ecclesiastica

Wearmouth/Jarrow
FAMILLE "WILLIBRORDIENNE"

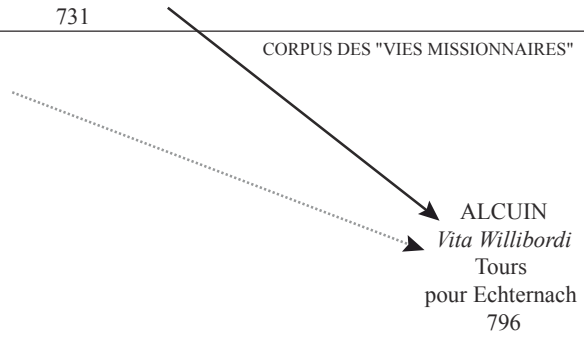

Figure 1 : Les deux « familles » de l'hagiographie missionnaire

(d'après I. N. Wood, The Missionary Life : Saints and the Evangelisation of Europe, 400-1050, Harlow, 2001, p. 52-53).

139. On prêtera une attention particulière aux textes insulaires qui, de la Navigatio Sancti Brendani au Voyage de Saint Brendan, opèrent la jonction entre cette littérature de l'exil et celle de l'« aventure » en utilisant le motif de longs voyages religieux sur la mer. S. SoвECKI, « From the désert liquide to the Sea of Romance : Benedeit's Le voyage de saint Brendan and the Irish immrama », Neophilologus, 87, 2003, p. 193-207 ; G. ConstABLE, « Monachisme et pèlerinage... », loc. cit., p. 13-14. 
Guénolé Ridoux, 8 hameau de Trébécourt, 02380 Jumencourt

\section{Vivre une métaphore : \\ écritures ango-saxonnes du voyage en mer au VIII ${ }^{\mathrm{e}}$ siècle}

Revisitant la question des origines du pèlerinage, des publications récentes ont rappelé qu'aux yeux des contemporains les premiers voyages religieux relevaient plutôt de l'expression d'une forme particulière de monachisme que d'une pratique de dévotion caractérisée par des rites et un réseau de destinations fermement institués. En examinant les sources du corpus des «Vies missionnaires », nous nous efforçons ici de montrer que les voyageurs religieux anglo-saxons des $\mathrm{VII}^{\mathrm{e}}$ et $\mathrm{VIII}{ }^{\mathrm{e}}$ siècles concevaient encore la peregrinatio pro Deo comme cette recherche d'un renoncement au monde matériel par le mouvement physique réel. La nécessité de justifier cette forme de dévotion, trop proche de l'errance des moines dénoncée par la Règle bénédictine, favorisa le recours au thème littéraire du voyage sur la mer, susceptible de décourager les critiques stigmatisant l'immoralité du genre de vie mené par les moines errants dans le monde séculier. L'aptitude du motif de la traversée maritime à se fondre dans la métaphore du chrétien-voyageur s'ajouta à une lecture « missionnaire » de l'histoire du peuple des Angles pour faire d'un voyage maritime initial une condition nécessaire de ce qui pouvait être considéré comme une pérégrination légitime. Ce thème littéraire, délaissé par l'hagiographie continentale au siècle suivant, imprima pourtant sa marque sur l'historiographie, et peut-être aussi sur la littérature, de la Bretagne insulaire.

Grande-Bretagne - Bède le Vénérable - hagiographie - peregrinatio - mer mission

\section{To Live a Metaphor:}

\section{Anglo-Saxon Writings about See Travels in the 8th Century}

While re-examining the questions of the origins of pilgrimage, recent publications suggested that contemporary observers considered the earliest religious travels more as another form of monasticism than as a religious practice characterized by a cluster of definite rituals and a network of distinct holy places. In this essay, using Ian Wood's work on missionary hagiography, we will argue that Anglo-Saxon religious travellers from the seventh and eighth centuries still considered the peregrinatio pro Deo as the pursuit of withdrawal from the material world through physical movement. In order to justify this particular kind of religious devotion, which was, to many extents, too close to the kind of wandering monasticism denounced by the Benedictine Rule, authors used and developed the literary theme of travel by water, which could discourage criticism of the corrupting way of life lived by wandering monks within the secular world. 
The matching of the sea travel theme with the metaphor of the Christian as a traveller added to a "missionary" conception of the gens Anglorum's history in establishing initial maritime journey as a necessary pre-condition of what could be properly considered as a peregrinatio by contemporary authors. The topic of water travel was comparatively neglected by continental authors of later missionary Lives. Nonetheless, it stamped its mark on early medieval Britain's historiography, and also, maybe, on its literature.

Britain - Bede - hagiography - peregrinatio - sea - mission 
